\title{
Impact of COVID-19 on thyroid cancer surgery and adjunct therapy
}

\author{
Sohail Bakkar ${ }^{1}$ ( Khaled Al-Omar $^{1} \cdot$ Qusai Aljarrah ${ }^{2} \cdot$ Moh'd Al-Dabbas $^{3} \cdot$ Nesrin Al-Dabbas $^{3} \cdot$ Samara Samara $^{3}$. \\ Paolo Miccoli ${ }^{4}$
}

Received: 24 May 2020 / Accepted: 10 June 2020 / Published online: 14 June 2020

(C) Italian Society of Surgery (SIC) 2020

\begin{abstract}
COVID-19 has profoundly modified the way healthcare is delivered. Jordan imposed lockdown and restrictive policies between March 17 and May 20, 2020. We aimed to assess the impact of such measures on thyroid cancer treatment plans. In the specified period, 12 patients were scheduled for surgery. Since papillary carcinoma was the preoperative diagnosis in all cases, radioactive iodine ablation (RIA) therapy was also planned 3-4 weeks following surgery after withdrawing thyroxine and achieving a thyroid stimulating hormone (TSH) level $>30 \mathrm{mU} / \mathrm{L}$. Thyroxine withdrawal is the routine method applied for RIA in Jordan as it is less costly compared to the rapid method of exogenous stimulation using recombinant TSH. All surgical procedures were performed without delay since all patients were asymptomatic per flu-like illness and came from a region of low COVID-19 prevalence. These included total thyroidectomy $(n=11)$, bilateral therapeutic central compartment neck dissection $(n=7)$, lateral compartment neck dissection $(n=5)$. However, the RIA treatment plan was altered considerably according to the period in which they were operated. 6 out of the 7 patients operated in March changed to the stimulated method of RIA at a considerable additional extra cost. The seventh patient and the April patient opt to delay RIA until after lockdown. The remaining cases (operated in May) followed the usual withdrawal method as restrictions were due to an end. The restrictive measures applied during COVID-19 did not affect the safe and timely delivery of surgical care. However, it added a financial and psychological burden to the entire cancer management plan.
\end{abstract}

Keywords COVID-19 $\cdot$ SARS-CoV-2 $\cdot$ Pandemic $\cdot$ Thyroid surgery

\section{Introduction}

The 2019 novel coronavirus and the disease it causes (COVID19) is a public health crisis that has profoundly modified the way medical and surgical care is delivered. Countries around the globe had a variable initial response to the COVID-19 pandemic from imposing massive lock downs and quarantine to surrendering to herd immunity. Globally, healthcare authorities have reacted by restricting medical care to emergency cases and postponing elective surgical procedures of all types.

Sohail Bakkar

sohail.bakkar@gmail.com

1 Department of Surgery, Faculty of Medicine, The Hashemite University, Zarqa 13133, Jordan

2 Department of Surgery, Faculty of Medicine, Jordan University of Science and Technology, Irbid 22110, Jordan

3 Farah Medical Campus (FMC), Amman 11183, Jordan

4 Department of Surgical, Medical, Pathology, and Critical Care, The University of Pisa, 56124 Pisa, Italy
The priority was made for the treatment of COVID-19 patients and emergency cases, in anticipation of a possible need for hospital beds and resources [1]. The American College of Surgeons (ACS) called to prioritize appropriate resource allocation as it relates to elective procedures and minimize the use of essential items required to care for patients [2]. Nevertheless, the unpredictability of the timeline of COVID-19 in the absence of effective drug treatments and vaccination, suggests that patients may be deprived of access to needed surgical care, likely for many months. The potential outcome of delay in necessary elective surgical procedures may have a more detrimental impact on patients' health compared to that of COVID-19 itself. Therefore, we have proposed a comprehensive strategy that allows for the safe and timely delivery of surgical care during COVID-19 and applied it institutionally. The strategy provides a safe balance between the prevention of COVID-19 transmission and the delivery of surgical care [3]. The aim of this retrospective cohort study was to assess the impact of the restrictive healthcare measures applied in 
the kingdom of Jordan in the time of COVID-19 on treatment plans for thyroid cancer patients.

\section{Materials and methods}

The kingdom of Jordan imposed a massive lockdown and applied healthcare restrictive policies between March 17 and May 20, 2020 in response to the COVID-19 pandemic. During this period, 12 patients were scheduled for thyroid surgery with or without selective compartment oriented therapeutic neck dissection as part of their thyroid cancer management plan. Since papillary thyroid carcinoma (PTC) whether obtained by fine needle aspiration cytology or core needle biopsy was the preoperative diagnosis in all cases, radioactive iodine ablation (RIA) therapy was also planned as an adjunct for thyroid remnant ablation as per current clinical practice guidelines [4]. In Jordan, the conventional method of RIA based on thyroid hormone withdrawal (THW) is the routine. RIA is administered 3-4 weeks following surgery after achieving a thyroid stimulating hormone (TSH) level $>30 \mathrm{mU} / \mathrm{L}$. This is because the traditional way is considerably less costly compared to the rapid method of exogenous stimulation using recombinant human TSH (rh-TSH) aka thyrotropin alpha [5]. Furthermore, medical insurance in Jordan whether governmental or private does not cover rh-TSH whose retail price is set at 1000 Jordanian Dinars (JOD) [6]. The clinical records of the study cohort were retrospectively reviewed. The primary outcome of interest was to assess the impact of restrictive policies applied for COVID-19 on safe and timely delivery of both surgery and its adjunct therapy (remnant ablation by means of RIA). Changes from the routine THW method for RIA and their financial and psychological impact were also reported. The psychological impact of undue delay in receiving RIA was assessed using the Hamilton anxiety rating scale (HAM-A) which is a clinicianrated evaluation whose purpose is to analyze the severity of anxiety [7]. The scale consists of 14 items, each defined by a series of symptoms, and measures both psychic anxiety (mental agitation and psychological distress) and somatic anxiety (physical complaints related to anxiety). Each item is scored on a scale of 0 (not present) to 4 (severe), with a total score range of 0-56, where $<17$ indicates mild severity, 18-24 mild-tomoderate severity, and 25-30 moderate-to-severe.

Data collection and analysis were performed according to the institutional guidelines and the ethical standards of the Helsinki Declaration.

\section{Results}

There were 9 females and 3 males (3:1 female-to-male ratio) with an average age of 56 years (range 33-80 years) and an average tumor size of $44 \mathrm{~mm}$ (occult- $80 \mathrm{~mm}$ ). The procedures performed included total thyroidectomy $(n=11)$, bilateral therapeutic central compartment neck dissection $(n=7)$, lateral compartment neck dissection $(n=5)$. One of the cases did not include a total thyroidectomy as it was a re-do surgery for recurrent PTC in the central and lateral neck proven biochemically, structurally, and histologically. None of the planned procedures were delayed as all patients were asymptomatic as per flu-like illness and came from a region of no or low COVID-19 prevalence adhering to the screening policy applied by the institute for surgical patients during COVID-19 [3]. However, RIA treatment plans were altered considerably according to the period in which the patient was operated on. 7 patients were operated in March, 1 patient in April and 5 patients in May. 6/7 (86\%) of patients operated in March changed to the stimulated method of RIA at a considerable additional personal extra cost. The seventh March patient and the April patient both opt to delay RIA until after lockdown. The remaining cases (operated in May) followed the usual withdrawal method as restrictions were due to an end. Accordingly, 50\% (6/12) of the study cohort were subject to an additional extra personal cost of 1000 JOD per patient. Furthermore, the additional delay in receiving conventional RIA experienced by 2 patients (17\%) placed them in the mild-to-moderate anxiety group according to the HAM-A scale [7].

\section{Discussion}

Although maintaining the infrastructure of healthcare systems and their capacity to accommodate a potentially overwhelming increase in critical patient care needs is important during a pandemic, providing safe and timely surgical care is considered equally important adhering to the motto of Medicine "primum non nocere" [3]. When prioritizing surgical cases, these fall along a continuum and are stratified according to indication and degree of urgency bearing in mind that many elective surgical procedures could upgrade in terms of priority at any time and become urgent or emergent [8]. Surgical treatment plans for cancer are considered as essential elective procedures [8]. Nevertheless, the cancer patient should be regarded as a person under a special and severe form of stress [9]. There is mounting anxiety which may at times increase to where the individual is disorganized [9]. Therefore, undue delay in delivering essential surgical care has the potential for an additive detrimental impact on mental health. Our institute's policy for the care of surgical patients in the time of COVID-19 dictates proceeding with essential elective cancer surgery in patients coming from areas of no or low regional prevalence without nucleic acid amplification testing [3]. Our results reflect the policy's effectiveness in allowing for the safe and timely delivery of essential elective surgical care. All 12 thyroid cancer surgeries were performed as scheduled and viral transmission 
was not manifested. However, the lockdown imposed by the government across international borders and, therefore, the limited availability of radioactive iodine, altered the method of delivering adjunct RIA therapy. 50\% of the study cohort were subject to a considerable additional financial burden at a time where financial resources are of utmost importance. Furthermore, those who could not afford an additional cost for therapy had to cope with an additional burden of stress.

This study is limited by its sample size and retrospective nature. Nevertheless, it highlights the importance of delivering safe and timely essential surgical care and its implications on both economic well-being and mental health.

\section{Conclusion}

In a region of no or low COVID-19 prevalence, restrictive policies applied did not affect the safe and timely delivery of surgical care for thyroid cancer patients. Nevertheless, adjunct therapy was subject to either an additional considerable financial burden or an undue delay with considerable implications on mental health.

\section{Data availability statement}

The data supporting the findings of this study are available from the corresponding author upon request.

Author contributions Corresponding author SB: study concept and design, data interpretation, drafting, final approval, and accountability for all aspects of the work. MA, NA: data analysis and interpretation, final approval, and accountability for all aspects of the work. KA, QA, SS and PM: critical revision, final approval, and accountability for all aspects of the work.

Funding This study was not funded by any grant.

\section{Compliance with ethical standards}

Conflict of interest The authors declare that they have no conflict of interest.
Research involving human participants or animals Ethical approval: All procedures performed in studies involving human participants were in accordance with the ethical standards of the institutional and/ or national research committee and with the 1964 Helsinki declaration and its later amendments or comparable ethical standards.

Informed consent Informed consent was waived because of the retrospective nature of this study.

\section{References}

1. Commins J (2020) Surgeon general urges providers to consider stopping all elective surgeries-hospitals push back. Health Leaders. 2020. https://www.healthleadersmedia.com/clinical-care/ surgeon-general-urges-providers-consider-stopping-elective-surge ries-hospitals-push. Accessed 17 Mar 2020

2. American College of Surgeons (ACS) (2020) COVID-19 update: guidance for triage of non-emergent surgical procedures. https:// www.facs.org/covid-19/clinical-guidance/triage. Accessed 17 Mar 2020

3. Al-Omar K, Bakkar S, Khasawneh L, Donatini G, Miccoli P (2020) Resuming elective surgery in the time of COVID-19: a safe and comprehensive strategy. Updates Surg. https://doi. org/10.1007/s13304-020-00822-6(ahead of print)

4. Miccoli P, Bakkar S (2017) Surgical management of papillary thyroid carcinoma: an overview. Updates Surg 69:145-150. https ://doi.org/10.1007/s13304-017-0449-5

5. Kowalska A (2015) Comparing the effectiveness of stimulation using rhTSH and thyroid hormone withdrawal in the treatment of thyroid cancer. Thyroid Res 8:A16. https://doi. org/10.1186/1756-6614-8-S1-A16

6. Fayed L (2020) Insurance coverage for Thyrogen. https://www. verywellhealth.com/insurance-coverage-for-thyrogen-514466. Accessed 5 May 2020

7. Hamilton $\mathrm{M}$ (1959) The assessment of anxiety states by rating. $\mathrm{Br}$ J Med Psychol 32:50-55

8. Stahel PF (2020) How to risk-stratify elective surgery during the COVID-19 pandemic? Patient Saf Surg 14:8. https://doi. org/10.1186/s13037-020-00235-9

9. Sutherland AM (1957) The psychological impact of postoperative cancer. Bull N Y Acad Med 33:428-445

Publisher's Note Springer Nature remains neutral with regard to jurisdictional claims in published maps and institutional affiliations. 